\title{
Study on the Effect of Atmosphere on A-type Zeolite Using Positron Annihilation Lifetime Technique
}

\author{
Hamdy F. M. Mohamed \\ Physics Department, Faculty of Science, El-Minia University,
}

The positron annihilation lifetime has been applied to study the effect of atmosphere (air and oxygen gas) on A-type zeolite (MS-4A). The measurements were performed at 1 atm of oxygen gas and at 1 atm of air media at room temperature. The lifetime spectra were analyzed using two methods: 1) average results of the ortho-positronium (o-Ps) lifetime and its intensity obtained by PATFIT program and 2) o-Ps lifetime and hole volume distributions given by Bayes' theorem and the maximum entropy principle (MELT program). The results showed that the o-Ps lifetime components have small values_when the sample was in oxygen gas medium compared with those in air medium and vacuum. This indicated that both the inhibition and quenching effects of the oPs parameters due to oxygen gas are taking place in MS-4A. 


\section{Introduction}

The behavior of positrons in condensed matter depends upon a variety of physical parameters, therefore this behavior is used to determine the properties of materials in which the positron annihilates. Depending upon the electronic structure of materials, the positron may remains free or combines remain free or combine with an electron to form positronium atom (Ps). The energetic positrons are thermalized in condensed matter (typically in 50-500 $\mu \mathrm{m})$ [1]. A free positron in a material could be annihilated via $2-\gamma$ mode with a rate, which is proportional to the electron density at the positron site; i.e. the positron could be used as a probe of the electron density at the annihilation site. Because two spin orientations are possible for an electron-positron pair, there are two ground states of Ps: 1). Spin-antiparallel para-positronium (p-Ps) has an intrinsic lifetime within $125 \mathrm{ps}$, decaying into two photons, with a formation probability of $25 \%$, 2) Spin-parallel ortho-positronium (o-Ps) annihilates into three photons with a theoretical lifetime of $140 \mathrm{~ns}$ in vacuum and a formation probability of $75 \%$ [2]. In condensed medium, however, the lifetime of o-Ps is shortened to few nanoseconds or less, because the positron in o-Ps annihilates with one of the bound electrons with opposite spin "Pick-off annihilation" [3]. In fact, the electron, positron and Ps are the only light particles participating in the low-energy physics and chemistry. Thus, tunneling, delocalization, and zero-point energy phenomena can play a great role in the electron, positron, and Ps behaviors because of their low mass quantum mechanical numbers.

Zeolite is known to be a kind of catalysis, catalyst, which has high catalystic activity due to its unique framework structure [4]. It is structurally unique in having cavities or pores with dimensions as a part of their crystalline structures and is found to have a wide application in industrial catalysts. catalysis. Bringing an aqueous salt solution in contact with the zeolite leads to incorporation of cations from the salt into the zeolite, replacing some of the non-framework cations initially presented. Ion exchange is the simplest and most important method for modifying the properties of zeolite.

Because to their increasing technological importance [5], zeolites came more and more into the focus of structural studies performed by a wide variety of methods. Among these it is by now well established that to certain questions (like the presence of free spaces in the structure, ionic contamination of internal surface, etc.) connected with many technological applications of zeolites (e.g., as host matrices for high dispersion metallic particles, catalysts, etc.,) the positron annihilation experiments may present adequate answers. Indeed, various types of zeolites were studied by the positron annihilation showing a correlation of the positron annihilation parameters with e.g. the cage size, the metallic ions present, the water contents, etc. [6-9]. In many of the previous 
studies of zeolites, effects of various absorbents on the positron annihilation lifetime parameters were examined $[10,13]$. But this involves a problem that the absorbent itself is reactive toward positron or positronium, which, combined with the inhomogeneous distribution of the absorbents introduces another complexity in interpreting the results. So, it is of interest to apply positron annihilation lifetime technique to study the effect of air and oxygen gas on the positron annihilation lifetime parameters in A-type zeolite.

\section{Experimental Set-up and Data Analysis}

The sample under investigation (A-type zeolite) is a pure powder without containing binder material, which is the product of Toyo Soda Kogyo Co., Japan. The basic structure of A-type zeolite is $\mathrm{SiO}_{4}$ and $\mathrm{AlO}_{4}$ groups in tetrahedral structure with either $\mathrm{Si}$ or $\mathrm{Al}$ at the center of the tetrahedron [14]. The $\mathrm{Al} / \mathrm{Si}$ ratio is equal to one and the $\mathrm{SiO}_{4}$ and $\mathrm{AlO}_{4}$ groups are joined together to form Archimedian truncated octahedron as shown in Fig. (1.a). Within the sodalite unit a void space ( $\beta$-cage) is existedexists with a maximum internal diameter of $0.66 \mathrm{~nm}$. To get into the $\beta$-cage, a molecule must pass through an opening $0.22 \mathrm{~nm}$ wide. The sodalite units are joined together along the cubic faces and the resulting arrangement creates a larger cavity (called $\alpha$-cage and its

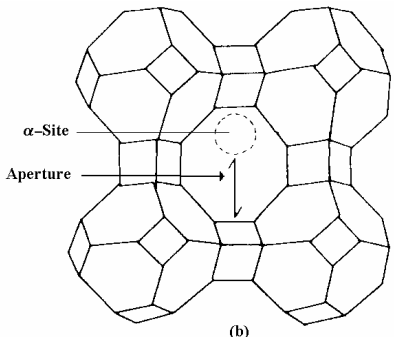

Fig. (1): A type zeolite:

a) The Archimedian truncated octaliedron or the sodalite unit.

b) The simple cubic array of truncated octahedron in the MS-4A. maximum diameter is $1.1 \mathrm{~nm}$ ), which is surrounded by eight sodalite units as shown in Fig. (1.b). To get into the $\alpha$-cage a molecule must pass through the aperture at the eight membered rings. Near this aperture there is a site, called $\alpha$-site, where a single charged cation could be accommodated. When $\mathrm{Na}^{+}$ion occupies this site, the diameter of the aperture is almost $0.4 \mathrm{~nm}$, and this is commercially named Molecular Sieve 4A (MS-4A).

The most commonly used positron source is ${ }^{22} \mathrm{Na}$, which is commercially available as aqueous solution of ${ }^{22} \mathrm{NaCl}$. The positron source was prepared by depositing about $20 \mu \mathrm{Ci}$ of aqueous ${ }^{22} \mathrm{NaCl}$ on a thin nickel foil of $2.2 \mathrm{mg} / \mathrm{cm}^{2}(2.5 \mu \mathrm{m})$ thick and area of $6 \times 6 \mathrm{~mm}^{2}$. After drying, it was covered with another foil of the same size. The source absorption by the nickel foil was about $6 \%$ and contributed to the short lifetime components. A conventional 
fast-fast coincidence spectrometer was employed for measuring the positron lifetime. The time resolution of the spectrometer was 290 ps (FWHM). The sample pellets (10 $\mathrm{mm}$ in diameter and $3 \mathrm{~mm}$ thickness) were formed from the pure powder of MS-4A under the pressure of 2 Tour. The samples were dried at $350^{\circ} \mathrm{C}$ for one hour in a vacuum chamber and the lifetime spectra for the sample under investigation were measured in vacuum, $1 \mathrm{~atm}$ air and $1 \mathrm{~atm}$ oxygen gas. All the positron annihilation lifetime measurements were performed at room temperature of about $25^{\circ} \mathrm{C}$.

The analysis of the positron annihilation lifetime spectra were performed using the PATFIT program [15] without source correction, which gives the average of the lifetimes and their intensities. Three lifetime components (in air medium) and four lifetime components (in vacuum and in oxygen gas medium) were found to give the best variance ratio and most reasonable standard deviations. Knowledge of $\tau_{3}$ or $\tau_{4}$ allows one to obtain the average radius $R$ of the nanoholes, in spherical approximation, from the TaoEldrup model $[16,17]$ :

$$
\tau_{4}^{-1} \text { or } \tau_{3}^{-1}=\lambda_{0}\left[1-\frac{R}{R_{0}}+\frac{1}{2 \pi} \sin \left(2 \pi \frac{R}{R_{0}}\right)\right],
$$

where; $\lambda_{0}=2 \mathrm{~ns}^{-1}, R_{0}=R+\delta R$, with $\delta R=0.1656 \mathrm{~nm}$ is the thickness of the homogenous electron layer in which the positron annihilates [18], from which we get the average volumes $v_{h}=4 \pi R^{3} / 3$. They should be interpreted as a rough estimate of the actual volumes, since real voids are not spherical, but irregularly shaped.

The positron annihilation lifetime spectra were also analyzed using Bayes' theorem and the maximum entropy principle. Deconvolutions were carried out through the computer code MELT [19]. The method employed for positron lifetime analysis in the program MELT (Maximum Entropy for Life Time analysis) has been described in some details [20-21]. This method of analysis gives additional information by calculating the probability density function of lifetime, $\operatorname{PDF}(\tau)=\lambda^{2} \alpha(\lambda)$, and distributions of the spectra.

\section{Results and Discussion}

Figure (2) shows the positron annihilation lifetime (PAL) spectra for the MS-4A in air, vacuum and oxygen gas. The shortest-lived and intermediate components are attributed mainly to the p-Ps and direct annihilation of positrons, respectively. Detailed analysis of these two components is difficult 
because of possible formations of positron and Ps compounds contributing to both of them. The short-lived components are not sensitive to the different surrounding media (air, vacuum and oxygen gas). The $3^{\text {rd }}$ and $4^{\text {th }}$ o-Ps components, which are related to the o-Ps in voids and the average of the o-Ps hole size $\left(\mathrm{V}_{\mathrm{h}}\right)$, that probed by the o-Ps lifetime $\left(\tau_{3}\right.$ or $\left.\tau_{4}\right)$, were calculated using Eqn. (1), and are summarized in Table (1).

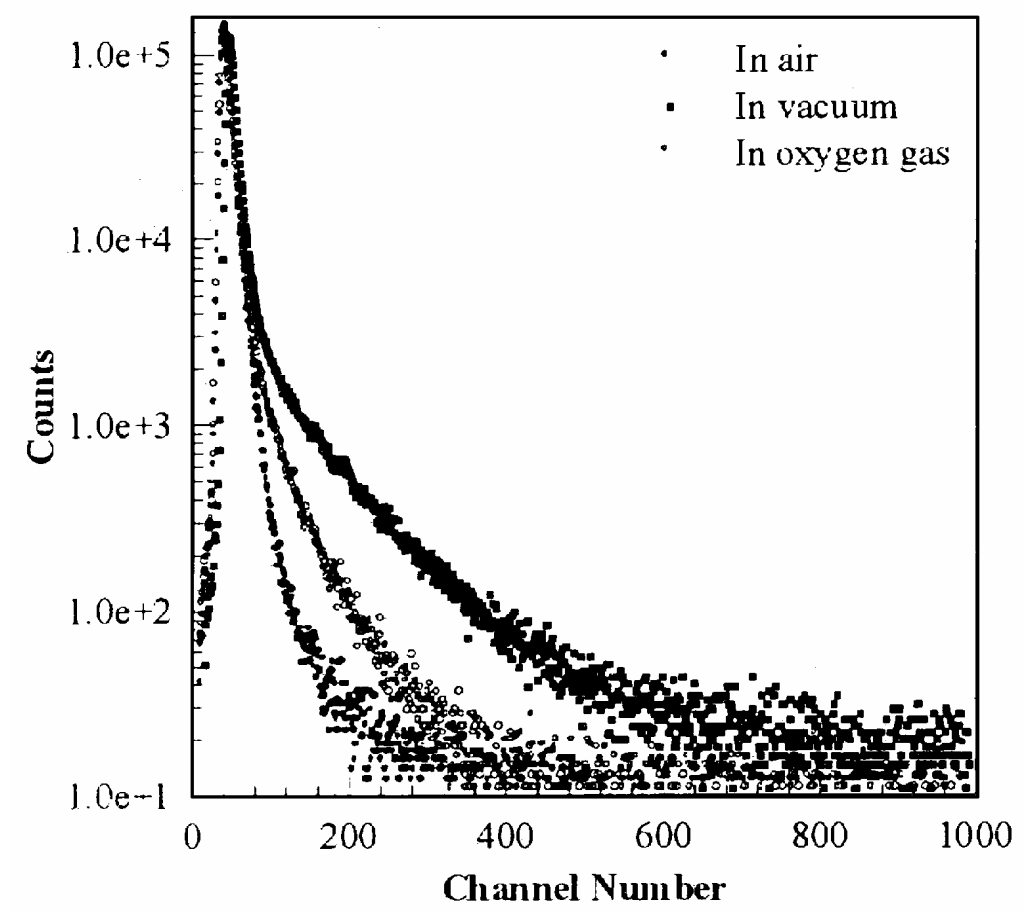

Fig. (2): Positron annihilation lifetime spectra for the MS-4A in air vacuum and oxygen gas

It is well known that the regions of smaller electron density are traps for positrons and Ps can be formed there. The presence of the long lifetimes $\left(\tau_{3}, \tau_{4}\right)$ gives a proof of Ps formation and these two components could be ascribed to pick-off annihilation of o-Ps in voids of the sample i.e., in $\alpha$-cage of MS-4A. There is a little probability that the long lived component $\left(\tau_{4}\right)$, for the MS-4A exposed to oxygen gas or in vacuum, may be due to the o-Ps on the surface of the MS-4A crystalline, since such surface should have longer lifetime and less intensity. As it is known, the $\beta$-cage in the MS-4A has a diameter of about 0.22 $\mathrm{nm}$ in which most gas molecules cannot come in while the $\alpha$-cage is large enough to hold a sphere with diameter of $1.1 \mathrm{~nm}$. Therefore, the $\alpha$-cage can accommodate small molecules such as $\mathrm{H}_{2}$ and $\mathrm{H}_{2} \mathrm{O}$, but the larger molecules are geometrically excluded. The molecular diameter of oxygen is $0.3 \mathrm{~nm}$ [22] and 
therefore it cannot get into the sodalite unit ( $\beta$-cage) of MS-4A at normal temperature but at higher temperatures, it becomes possible for the oxygen atom to overcome the activation barrier to enter the $\beta$-cage.

o-Ps hole volume $\left(\mathrm{nm}^{3}\right)$

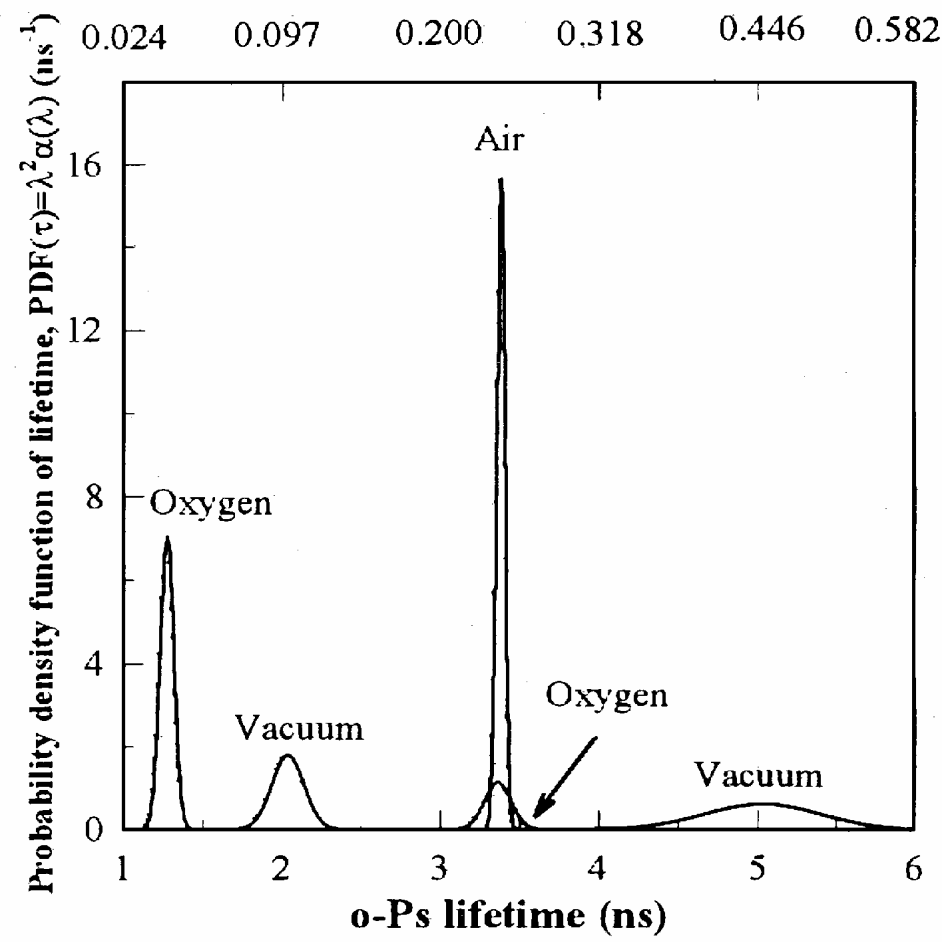

Fig. (3) The probability density function of lifetime as a function of the o-Os lifetime for the MS-4A in air, vacuum and oxygen gas. The scale at the top ordinate is the size of the hole in which the o-Ps is trapped

From Table (1) it is clear that the long-lived components for MS-4A in oxygen gas medium are smaller than those in vacuum. This is because the oxygen gas in the $\alpha$-cage decreases the intensities $\mathrm{I}_{3}$ and $\mathrm{I}_{4}$ (inhibition effect) and also decreases the lifetimes $\tau_{3}$ and $\tau_{4}$ (quenching effect) that is in a good agreement with the data obtained by Ito et al. [6]. They measured the lifetime at lower oxygen pressure of MS-5A zeolite and found that the o-Ps lifetime and its intensity are decreasingdecrease with the oxygen pressure. This indicates that both the inhibition and quenching effects are taking place in MS-5A as in MS4A. This is to be compared with the well recognized fact in liquid phase Ps chemistry that the oxygen quenches the o-Ps lifetimes, but does not inhibit Ps 
formation [23]. The change caused by different gases is of a minor effect compared to oxygen gas, which has paramagnetic property [8].

The most striking systematic effect is that oxygen gases reduce the influence on the MS-4A by localizing in the $\alpha$-cage, but its effect is complicated [6]. The effect of oxygen is mainly chemical reaction (spin conversion or oxidation). In gases, the quenching by oxygen is mainly by the spin conversion with paramagnetic oxygen [24] as:

$$
\text { o-Ps }+\mathrm{O}_{2} \rightarrow \text { p-Ps/o-Ps }+\mathrm{O}_{2} \text {. }
$$

In liquid, however, the quenching occurs mostly by chemical reaction [25] as:

$$
\mathrm{o}-\mathrm{Ps}+\mathrm{O}_{2} \rightarrow \mathrm{PsO}_{2}
$$

In the MS-4A, the spin conversion does not seem to be involved because the intensity of the p-Ps component does not show any increase by oxygen. The quenching of o-Ps in the voids of MS-4A is undoubtedly due to chemical reaction like reaction (3).

The long-lived component $\left(\tau_{4}\right)$ for the MS-4A exposed to airwas disappeared while the intensity of the o-Ps $\left(\mathrm{I}_{3}\right)$ is the smallest one. This may be attributed to the reaction of the Ps with different gasses in air that localized in the cage, which is agreedagrees with the data of Chen et al. [8]. The o-Ps parameters of the longest lived components for the MS-4A in vacuummedia are the largest oneas compared to theothers data in air and oxygen gas media. This may be due to the removeremoval of gas and water molecules absorbed in the cage of zeolites and the free volume between small zeolite grain particles.

Also, the positron annihilation lifetime spectra for the sample in different atmospheres have been analyzed using MELT program. The probability density function of lifetime, $\operatorname{PDF}(\tau)=\lambda^{2} \alpha(\lambda)$, as a function of the oPs lifetime for the MS-4A exposed to air, oxygen gas and vacuum are obtained and presented in Fig. (3). The scale at the top ordinate is the size of the hole in which the o-Ps is trapped (calculating (calculated using Eq. (1)). The average values of o-Ps lifetime components from MELT program are almost the same as those values from PATFIT program. The probability density function of o-Ps lifetime is wider and only one distribution for the MS-4A in air results, whereit is became the two peaks are broader and shifted to lower and higher values of the o-Ps lifetime. Peaks shift to the higher values indicatingindicates clearly that the size of the o-Ps hole volume becomes bigger aswhen the sample is put in vacuum atmosphere. On the other hand, for the MS-4A in oxygen gas the probability density function of the o-Ps lifetime washas two wider peaks when 
compared to thosese two peaks in vacuum where the first peak is shifted to shorter o-Ps lifetime (smaller o-Ps hole volume) and the other one is still in the same position as in air atmosphere. Also, the area under the two peaks of MS$4 \mathrm{~A}$ in vacuum is smaller than thatarea on MS- of MS-4A in oxygen gas. In case of air, the o-Ps distribution of MS-4A has only one long lived component and the area under this distribution is very high large.

\section{Conclusion}

Positron annihilation lifetime has been employed to investigate the sub-nm vacancies of MS-4A zeolite exposed to air and oxygen gas. These results indicate that the oxygen gas in MS-4A decreases both the o-Ps lifetime and its intensity (quenching and inhibition effects). Also, the reaction between the oxygen gas and Ps may be due to chemical reaction not spin conversion. The results also reflect the importance of using MELT program to extract the oPs lifetime distributions.

\section{References}

1. J. DeVries, Ph.D. thesis, "Positron lifetime technique with applications in materials science", Delftse Universit aire Pers, The Netherlands (1987).

2. R. N. West "Positron Studies of Condensed Matter", Barnes and Noble, New York (1974).

3. J. H. Lind, P. L. Jones, and G. Pearsall, J. Poly. Sci. A 24, 3033 (1986).

4. J. W. Ward, J. Catal. 9, 225 (1967).

5. D. W. Breck, "Zeolite molecular sieves, structure, chemistry and use"Molecular Sieves, Structure, Chemistry and Use" Wiley, New York (1974).

6. Y. Ito, T. Tanaka, and M. Hasegawa, Appl. Phys. A45, 193 (1988).

7. Y. Ito, M. Hirose, T. Tabata, and M. Hasegawa, Appl. Phys. A50, 39 (1990).

8. Z. Q. Chen, Z. Tang, and S. J. Wang, Mat. Sci. Forum 175-178, 667 (1995).

9. M. Hasegawa, Y. Tsuchiva, Y. Kitayama, T. Chiba, M. Saitoh, and S. Yamaguchi, Mat. Sci. Forum 105-110, 257 (1992).

10. M. B. Perkal and W. B. walters, Walters, J. Chem. Phys. 53, 190 (1970).

11. A. D. Mokrushin, A. O. Tatur, and V. P. Shantarovich, Izv. Akad. Nauk SSSR, Ser. Khim. 6, 1216 (1973).

12. H. Schut, G. R. Escobar, X. I. Kolar, A. Van Veen, and G. Clet, Radiation Phys. Chem. 58, 715 (2000). 
13. K. Suvegh, G. Vanko, A. Domjan, and A. Vertes, presented in $12^{\text {th }}$ Inter. Conf. on Positron Annihilation (ICPA-12), August 6-12, 2000 (München, Germany).

14. T. B. Reed and D. W. Breek, J. Am. Chem. Soc. 78, 5972 (1956).

15. P. Kirkegaard, M. Eldrup, O. E. Mogensen, and N. Pedersen, Comput Phys. Commun. 23, 307 (1981) and PATFIT 88 (1988).

16. S. J. Tao, J. Chem. Phys. 56, 5499 (1972).

17. M. Eldrup, D. Lightbody, and J. N. Sherwood, Chem. Phys. 63, 51 (1981).

18. H. Nakanishi, S. J. Wang, and Y. C. Jean, in "Positron annihilation studies of fluids",Annihilation Studies of Fluids", Sharama, S. C. (Ed.), World Scientific, Singapore, p.292 (1988).

19.A. Shukla, M. Peter, and L. Hoffmann, Nucl. Instr. Meth. A335, 310 (1993).

20.A. Shukla, L. Hoffmann, A. A. Manuel and M. Peter, Mater. Sci. Forum 175-178, 939 (1995).

21.L. Hoffmann, A. Shukla, M. Peter, B. Barbiellinni and A. A. Manuel, Nucl. Instr. Meth. A335, 276 (1993).

22.A. Bondi "Physical properties of molecular crystals, liquids anf glasses",Properties of Molecular Crystals, Liquids and Glasses", Wiley, New York (1968).

23.A. M. Cooper, G. J. Laidlaw, B. G. Hogg, J. Chem. Phys. 46, 2441 (1967).

24.R. L. Klobuchar, P. J. Karol, J. Phys. Chem. 84, 483 (1980).

25.J. Lee, G. J. Celitans, J. Chem. Phys. 44, 2506 (1966). 